Special Issue of the 7th International Advances in Applied Physics and Materials Science (APMAS 2017)

\title{
Mechanical Properties of Nanoporous Si Anodes using a Continuum Mechanical Model
}

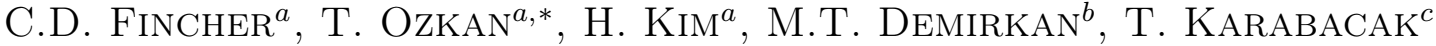 \\ AND A.A. POLYCARPOU ${ }^{a}$ \\ ${ }^{a}$ Department of Mechanical Engineering, Texas A\&M University, College Station, TX, 77843, USA \\ ${ }^{b}$ Department of Materials Science and Engineering, Gebze Technical University, Gebze, Kocaeli, 41400, Turkey \\ ${ }^{c}$ Department of Physics \& Astronomy, University of Arkansas at Little Rock, Little Rock, AR 72204, USA \\ Silicon $(\mathrm{Si})$ electrodes possess a theoretical specific capacity nearly ten times that of current graphite electrodes \\ used in lithium ion batteries. However, lithiation and delithiation induce large volume changes within the Si, \\ resulting in cracking and eventual capacity loss with cycling. Recent experimental evidence indicates that the \\ presence of nanoporosity may mitigate capacity fade. By implementing a scalable differential effective medium \\ approach, we elucidate the effects of nanoporosity upon the mechanical properties of fully-lithiated amorpohous Si \\ anode films. Our analytical findings suggest that increased pore volume fraction significantly alters the mechanical \\ properties of nanofilms and enhances anode survivability. Meanwhile, the auxetic limit imposes an upper bound on \\ porosity specific fracture toughening. Overall, the results of this paper provide design guidelines for multilayered \\ nanoporous Si thin films with increased capacity retention.
}

DOI: 10.12693/APhysPolA.134.401

PACS/topics: lithium-ion battery, silicon anode, thin film, nanomechanics, capacity fade

\section{Introduction}

Lithium ion batteries (LIBs) can provide superior energy density per unit mass and volume compared to other types of rechargeable batteries. However, conventional graphite electrode materials require large anodes in order to meet the ever-increasing demands of portable battery systems. As a result, recent research has focused on the development of $\mathrm{Si}$ based electrodes. Si electrodes have a high theoretical gravimetric capacity of around $4200 \mathrm{mAh} / \mathrm{g}$, approximately ten times the gravimetric capacity of current graphite electrodes. However, high capacity anodes suffer from serious irreversible capacity drop and poor cyclability due to huge volume changes associated with $\mathrm{Li}+$ insertion and extraction. For example, as lithium is inserted, Si electrodes swell approximately $300 \%$, resulting in large strains [1]. Over the course of many cycles, this swelling leads to decreased capacity as a result of cracking, pulverization, and loss of active material. This mechanism of capacity fade, often referred to as decrepitation, is among the most severe obstacles to realization of high capacity next-generation silicon anodes in rechargeable LIBs.

Recently, nanostructuring of the anode material has successfully mitigated some issues associated with capacity fade and decrepitation. For example, anodes can be structured in composite form, with an inactive and compliant or hollow space which is built to accommodate large volume change associated with active material swelling $[2,3]$. Providing further analysis,

* corresponding author; e-mail: tozkan@tamu.edu
Haftbaradaran et al. studied the critical film thickness to avoid fracture and delamination of Si electrodes, thus reducing the severity of decrepitation [4]. More recently, Ma et al. identified the necessary conditions for averting lithiation-induced fracture of $\mathrm{Si}$ anodes in terms of the critical size and state of charge of Si by computationally employing the bond order-length-strength (BOLS) mechanism [5]. Ma et al. observed strong critical sizeshape correlation for complete fracture resistance during lithiation and predicted the maximum value of the characteristic length for a mechanically stable nanostructured Si anode to be $\approx 90 \mathrm{~nm}$ for nanoparticles, $\approx 70 \mathrm{~nm}$ for nanowires and $\approx 33 \mathrm{~nm}$ for nanoscale thin films [5].

However, several experimental studies suggest that the incorporation of nanoporosity significantly affects the diffusion properties in $\mathrm{Si}$ [6]. Moreover, the insertion and extraction of $\mathrm{Li}+$ in an electrode is often modeled as the diffusion of interstitial atoms at the continuum level [711]. Due to the high porosity, low density thin films can enhance the mechanical properties of films by accomodating the volumetric changes accompanying lithium insertion and removal. Thus, optimization of the density and the porosity of thin films may enhance mechanical survivability and electrochemical performance of Si thin film anodes [12,13]. However, the application of a continuum mechanical model may better elucidate the fracture toughening directly attributable to nanoporosity.

A recent study published by Ozkan et al. dealt with the effects of nanoporosity in the elastic and plastic response of density modulated nanoporous tungsten thin films, a material which is assumed nanoporous and nearisotropic [14]. However, because recently published elastic constants for amorphous fully-lithiated Si reveal the dilatation-corrected Zener ratio to be near unity, a similar 
scalable differential elastic medium approach (SDEMA) based model may elucidate the elastic and plastic response of fully lithiated $\mathrm{Si}[15]$. In response, the primary objective of this paper is to estimate the effect of nanoporosity upon the predicted critical film thickness to avoid lithiation-induced fracture in Si LIB anodes.

\section{Methods}

We extend the SDEMA to the fracture energy release model used by Ma et al. to determine the critical thickness to avert lithiation-induced fracture of nanoporous Si anodes in LIBs. Previous research has shown that the adjustment of deposition parameters allows sputter coating of thin films with tunable morphology, as explained in the structural zone model [16, 17]. A simple way to change the density of the film is to change the deposition gas pressure during growth process, where high gas pressure provides low density thin films and vice versa. Thus, variation of the working gas pressure during sputtering enables the fabrication of thin films with tunable density and porosity, with pore sizes ranging from several $\mathrm{nm}$ to $\AA[13,16,18]$. In our analysis, we assume an average pore size of $2-3 \mathrm{~nm}$ for density modulated Si thin films, as shown in Fig. 1 [13].

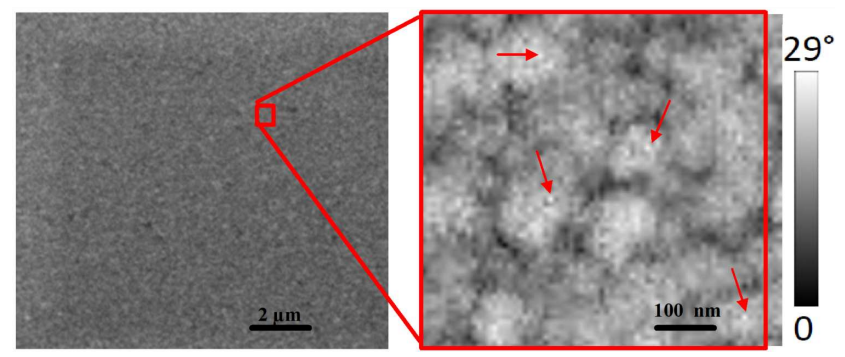

Fig. 1. Left: SEM image of a density modulated Si thin film sample with $\approx 21 \%$ porosity content grown using the method described in detail in [13]. Right: nanoFTIR phase image of the area indicated with the red box on the left. Signal attenuation at the boundary of nanopores results in contrast enhancement allowing for the detection of pore size. The maximum pore size appears to be around $5 \mathrm{~nm}$ as shown with the red arrows.

From a purely fracture mechanics perspective, the presence of nanoporosity should enhance durability and fracture toughness of the resulting thin film, as the pores distort the continuity of the stress field by generating traction free surfaces. As a results, the pores will either arrest the crack, or force the crack to crawl around the pore prior to global failure. Additionally, upon full lithiation, the pores accommodate some of the silicon expansion, thus reducing crack driving forces [13].

From Ma et al. work, the minimimum thickness to avert lithiation-induced fracture in nanofilms can be written as follows, assuming the film as rigid-plastic [5]:

$$
h_{c}=\frac{\Gamma E_{b}+\sqrt{\Gamma^{2} E_{b}^{2}+4 Z \sigma^{2} \Gamma E_{b} c \tau d_{0}}}{8 Z \sigma^{2} \mathrm{SOC}},
$$

where $E_{b}$ represents the Young modulus of the bulk material, $\sigma$ is the representative stress and $\sigma=\sigma_{y}, \Gamma$ represents fracture energy, $d_{0}$ is bond length, $Z$ is an order based upon fracture geometry which is on the order of unity, SOC represents state of charge, and $\tau$ represents a shape factor. Meanwhile, $c$ represents surface bond strength, which can be modeled as follows [19]:

$$
\begin{aligned}
& c=\sum_{i \leq 3} c_{i}\left[c_{i}^{-(m+3)}-1\right], \\
& c_{i}=2\left[1+\exp \left(\frac{12-z_{i}}{8 z_{i}}\right)\right]^{-1},
\end{aligned}
$$

where $c_{i}$ represents the bond contraction coefficient and $z_{i}$ is the effective coordination number of the atom of concern. For amorphous silicon, $c$ can be calculated as approximately 1.875 through Eqs. (2) and (3), with $m$ as 4.88 [19]. For thin film geometry, representative values of $\Gamma=10\left[\mathrm{~J} / \mathrm{m}^{2}\right][20], Z=0.91[21]$, and $\tau=1$ for a nanoscale film [5]. Meanwhile, for amorphous silicon, the bond length, $d_{0}=0.278 \mathrm{~nm}$ [5].

However, in the case of many nanoporous and nanoarchitectured films, mechanical analysis becomes significantly more complicated, as $\sigma, \nu$, and $E$ are all functions of porosity.

For sputter-deposited nanoporous tungsten thin films, Ozkan et al. showed the dependence of Young's modulus and Poisson's ratio upon porosity packing fraction $\varphi$ as follows in Eqs. (4) and (5) [14]:

$$
\begin{aligned}
& E_{f}(\varphi)=E_{0}\left(1-\frac{\varphi}{\varphi_{\max }}\right)^{\frac{23 \varphi_{\max }}{12}}, \\
& \nu_{f}(\varphi)=\left(1+\nu_{0}\right)\left(1-\frac{\varphi}{\varphi_{\max }}\right)^{\frac{\varphi_{\max }}{4}}-1,
\end{aligned}
$$

where $E_{f}$ and $\nu_{f}$ represents the effective Young modulus and Poisson ratio of the architecture nanofilm, and $E_{0}$ and $\nu_{0}$ represent the Young modulus and Poisson ratio of the parent materials without porosity. $\varphi_{\max }$ is the maximum packing volume fraction of the pores, in this case 0.637 [14].

Additionally, Johnson [22] showed $\sigma$ as a function of porosity as follows:

$$
\sigma(\varphi)=\frac{\cot \alpha E(\varphi)}{6(1-\nu(\varphi)) \exp \left(\frac{3 \kappa}{2}-2\right)-4(1-2 v(\varphi))},
$$

where $\alpha$ represents the angle of the indenter tip, where $\kappa$ represents the relationship between hardness $H$ and stress, given in Eq. (7):

$$
\kappa=\frac{H(\varphi)}{\sigma(\varphi)}
$$

The Young modulus and the Poisson ratio for non-porous amorphous and lithiated silicon have previously been estimated as $E_{0}=41 \mathrm{GPa}$ and $\nu_{0}=0.25$ [15], where $H$ has been previously found as $1.3 \mathrm{GPa}[23]$. By solving Eqs. (7) and (6) iteratively, using $\varphi$, as zero and solving iteratively, $\kappa$ can be found as 2.56 , which agrees well with other porous thin films [24]. 
By using $\kappa$ as 2.56, we solve Eqs. (1), (6), and (7) over a range of porosity values, we find critical film thickness to avoid lithiation-induced fracture as a function of porosity. Similarly the application of Eqs. (4), (5), and (6) allow the determinaton of the Young modulus, the Poisson ratio, and yield strength at full lithiation as a function of porosity, as seen in Figs. 2 and 3.

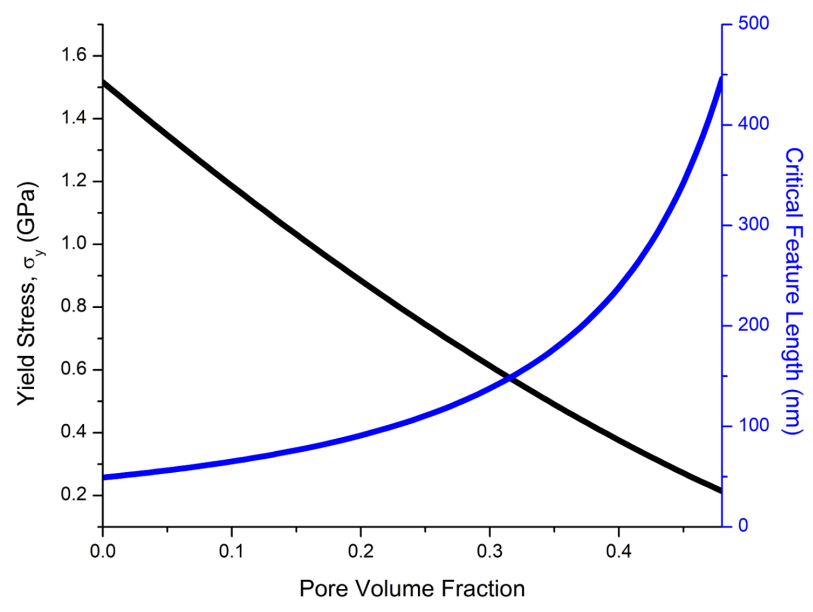

Fig. 2. Yield stress and critical feature length versus pore volume fraction.

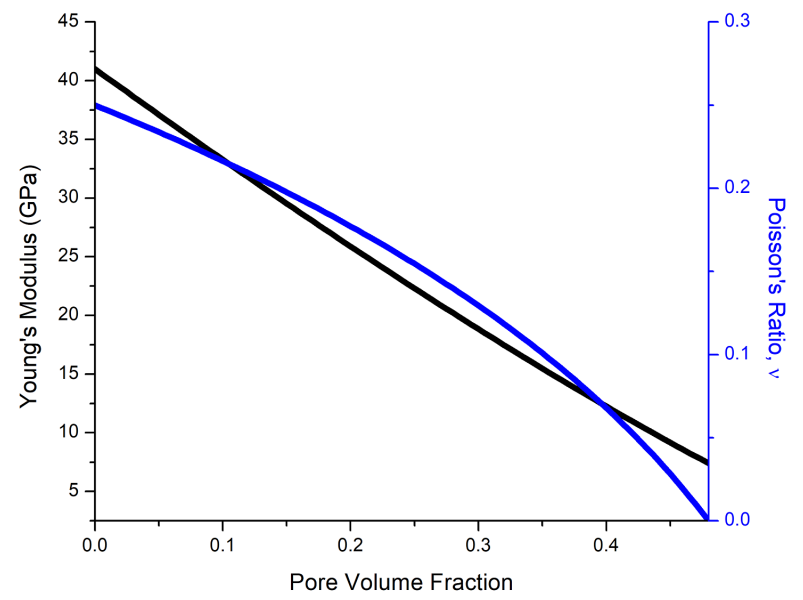

Fig. 3. The Young modulus and the Poisson ratio versus pore volume fraction.

\section{Results}

As seen in Fig. 2, the auxetic limit of $48 \%$ pore volume fraction represents the limit of pore specific fracture toughening. Over the range of pore volume fractions studied, film thicknesses less than the predicted critical feature length are required to prevent fracture. Thus, the minimum film thickness to avert lithiation-induced fracture increases with pore volume fraction. For films of $20 \%$ porosity, as studied by Demirkan et al., a film thickness of approximately $90 \mathrm{~nm}$ or less is required to prevent lithiation-induced fracture completely, nearly double the required thickness of $49 \mathrm{~nm}$ for a nonporous monolithic thin film. However, even approaching the auxetic limit, the critical thickness to avert lithiatiation-induced fracture fails to exceed several hundred nm. Thus, fracture aversion through nanoporosity alone would not suffice to reach industrially significant thicknesses in the $\mu \mathrm{m}$ range. Conceivably, the implementation of a multilayered nanoporous thin film architecture has potential to bypass limitations experienced by monolithic films including decrepitation and capacity fade.

As seen in Fig. 3, the Poisson ratio exhibits significantly more sensitivity compared to elastic modulus as the porosity approaches the auxetic limit. This suggests that the Poisson ratio may play a dominant role in the phenomenon of disintegration of highly porous films $[13,16]$. As a result, porosity greater than $40-45 \%$ volume fraction may no longer enhance fracture mitigation properties.

\section{Conclusions}

Within this study, we have outlined the critical film thickness to avert lithiation-induced fracture within amorphous silicon thin films. We find that porosity alone does not suppress lithiation-induced cracking enough to allow the design of durable single-layer battery anodes with $\mu \mathrm{m}$-level thickness. However, our model may inform about the design of multilayer nanoporous anodes. Thus, further studies should be conducted upon conditions required for the degradation and delamination of multilayered nanoporous film architectures.

We acknowledge that this study also has limitations. For example, we observed that $\kappa$ is highly sensitive to porosity. As a result, experimental testing should be devoted to better characterize $\kappa$ at various pore volume fractions, with $H$ being measured for a sample with a more rigorously characterized porosity. Additionally, the pre-factor $Z$ is known to vary with system geometry and amount of plasticity. Thus, we hope our study may motivate further experimentation to elucidate the effects of nanoporous structure upon the pre-factor and thus the critical feature length for anode architectures. Lastly, we assume uniform pore size of $2-3 \mathrm{~nm}$. However, the effects of different pore diameter are not thoroughly explored. Because the degree of toughening may vary with pore diameter, the usage of X-ray porosimetry in combination with mechanical testing may further validate the effects of feature and pore size upon anode fracture toughening.

\section{References}

[1] M.N. Obrovac, L. Christensen, Electrochem. Solid State Lett. 7, A93 (2004).

[2] H. Wu, G. Zheng, N. Liu, T.J. Carney, Y. Yang, Y. Cui, Nano Lett. 12, 904 (2012).

[3] K. Zhao, M. Pharr, L. Hartle, J.J. Vlassak, Z. Suo, J. Power Sourc. 218, 6 (2012).

[4] H. Haftbaradaran, X. Xiao, M.W. Verbrugge, H. Gao, J. Power Sourc. 206, 357 (2012).

[5] Z. Ma, T. Li, Y.L. Huang, J. Liu, Y. Zhou, D. Xue, RSC Adv. 3, 7398 (2013). 
[6] D. Kondrashova, A. Lauerer, D. Mehlhorn, H. Jobic, A. Feldhoff, M. Thommes, D. Chakraborty, C. Gommes, J. Zecevic, P. de Jongh, A. Bunde, J. Kärger, R. Valiullin, Sci. Rep. 7, (2017).

[7] R.E. Garcia, Y.M. Chiang, W.C. Carter, P. Limthongkul, C.M. Bishop, J. Electrochem. Soc. 152, A255 (2005).

[8] J. Christensen, J. Newman, J. Solid State Electrochem. 10, 293 (2006).

[9] J. Christensen, J. Newman, J. Electrochem. Soc. 153, A1019 (2006)

[10] X. Zhang, W. Shyy, A.M. Sastry, J. Electrochem. Soc. 154, A910 (2007).

[11] Y.-T. Cheng, M.W. Verbrugge, J. Power Sourc. 190 453 (2009).

[12] M. Demirkan, L. Trahey, T. Karabacak, J. Power Sourc. 273, 52 (2015).

[13] M. Demirkan, L. Trahey, T. Karabacak, Thin Solid Films 600, 126 (2016).

[14] T. Ozkan, M.T. Demirkan, K.A. Walsh, T. Karabacak, J. Mater. Res. 31, 2011 (2016).
[15] Y. Qi, L.G. Hector, C. James, K.J. Kim, J. Electrochem. Soc. 161, F3010 (2014).

[16] R. Messier, A.P. Giri, R.A. Roy, J. Vac. Sci. Technol. A 2, 500 (1984).

[17] J. Thornton, J. Vac. Sci. Technol. 11, 666 (1974).

[18] D. Ren, Y. Zou, C. Zhan, N. Huang, J. Kor. Phys. Soc. 58, 883 (2011).

[19] C.Q. Sun, Phys. Rev. B 69, 045105 (2004).

[20] K. Zhao, M. Pharr, J.J. Vlassak, Z. Suo, J. Appl. Phys. 109, 016110 (2011).

[21] K. Zhao, M. Pharr, Q. Wan, W.L.W.E. Kaxiras, J.J. Vlassak, Z. Suo, J. Electrochem. Soc. 159, A238 (2012).

[22] K.L. Johnson, Contact Mechanics, Cambridge University Press, 1985.

[23] L.A. Berla, S.W. Lee, Y. Cui, W.D. Nix, J. Power Sourc. 273, 41 (2015).

[24] Y. Ding, Z. Zhang, in: Handbook of Nanomaterials, Springer, New York 2013, p. 799. 